\title{
O EFEITO AGUDO DO TREINO DE ALONGAMENTO ESTÁTICO DOS MÚSCULOS ÍSQUIOS-TIBIAIS NA AGILIDADE DO TENISTA
}

\author{
Marcos Aurélio Borges Muniz, Universidade do Contestado - UnC, Santa Catarina - \\ Brasil \\ Luis Paulo Gomes Mascarenhas, Universidade do Contestado - UnC, Santa Catarina - \\ Brasil \\ Marcos Tadeu Grzelczak, Universidade do Contestado - UnC, Santa Catarina - Brasil \\ Wallace Bruno de Souza, Universidade do Vale do Itajaí - Univali, Santa Catarina - \\ Brasil \\ Camila Pedrassani, Universidade Federal do Paraná - UFPR, Curitiba, Paraná - Brasil \\ William Cordeiro de Souza, Universidade do Contestado - UnC, Santa Catarina - Brasil \\ Sérgio Dimas de Paula, Universidade do Contestado - UnC, Santa Catarina - Brasil \\ Valderi Abreu de Lima, Universidade do Contestado - UnC, Santa Catarina - Brasil
}

\section{RESUMO}

O presente estudo buscou como objetivo avaliar o efeito agudo do treino de alongamento estático dos músculos ísquios-tibiais na agilidade do tenista. A amostra foi composta por 10 tenistas que praticam o esporte Tênis de Campo três vezes por semana, duas horas por dia, com média de idade de 32,8 $\pm 2,09$ anos, peso 83,7 \pm 7,76, estatura 1,82m $\pm 6,74$ e Índice de Massa Corporal (IMC) de 25,18 $\pm 1,78$. Foi realizado o teste de elevação de membro inferior estendido de Kendall, para detectar o encurtamento dos músculos ísquiostibiais. O ângulo médio foi obtido na flexão da perna estendida em direção à pelve do membro inferior direito e esquerdo. Para avaliação de pré e pós-teste de agilidade foi aplicado o teste de Shuttle-Run. Na análise dos dados foi utilizado à estatística descritiva: média, desvio padrão (dp), frequência de percentual (\%) e o teste t de student com nível de significância de 0,05 . Foi observado que houve uma diferença significativa nos testes de agilidade antes e pós-alongamento estático. Assim, foi possível verificar uma melhora de 17\% na média do desempenho do teste de agilidade-com a aplicação do treino de alongamento estático dos músculos ísquios-tibiais.

Palavras-Chave: Tenistas, Alongamento estático, Agilidade.

\section{THE ACUTE EFFECTS OF STATIC STRETCHING WORKOUT HAMSTRINGS MUSCLES IN THE LEGS OF TENNIS}

\begin{abstract}
This study aimed to evaluate the acute effects of static stretching workout hamstrings muscles in the legs of tennis. The sample consisted of 10 tennis players who practice the sport of Tennis Field three times a week, two hours per day, with a mean age of $32.8 \pm$ 2.09 years, weight $83.7 \pm 7.76$, height $1,82 \mathrm{~m} \pm 6.74$ and Body Mass Index (BMI) of 25.18 \pm 1.78 . Lift the extended leg Kendall test was performed to detect the shortening of the hamstrings muscles. The average angle was obtained in flexion of extended toward the pelvis of the right leg and left leg. To review pre and post-test agility of the Shuttle-Run Test was applied. Mean, standard deviation (SD), frequency percentage (\%) and the


Student $t$ test with significance level of 0.05 . In the data analysis descriptive statistics were used. It was observed that there was a significant difference in the agility tests before and after static stretching. Thus, we observed an improvement of $17 \%$ on the average performance of the agility test, with the implementation of training static stretching of the hamstrings muscles.

Key-Words: Tennis players, Static stretching, Agility.

\section{EI EFECTO AGUDO DEL ENTRENAMIENTO DE ELONGACION ESTATICODE LOS MUSCULOS ISQUIOTIBIALES EN LA AGILIDAD DEL TENISTA}

\section{RESUMEN}

El estudio tuvo como objectivo evaluar los efectos agudos del estiramiento estático de los músculos isquiotibiales em la agilidad del tenista. La muestra estuvo conformada por 10 jugadores de tenis que practican el deporte del tenis de campo, tres veces a la semana, dos horas por día, con una edad media de 32,8 $\pm 2,09$ años, peso $83,7 \pm 7,76$, altura $1,82 \mathrm{~m} \pm$ 6,74 y el índice de masa corporal (IMC) de $25,18 \pm 1,78$. Se realizó el teste de la pierna extendida (prueba de Kendall) para detectar el acortamiento de los músculos isquiotibiales. El ángulo promedio se obtuvo en la flexión de la pierna extendida hacia la pelvis de la pierna derecha y la izquierda. Para evaluación pre y agilidad post-test de la Prueba de Shuttle-Run se aplicó. Media, desviación estándar (DE), el porcentaje de frecuencia (\%), y la prueba t de Student con un nivel de significación de 0,05. Se utilizó el análisis de los datos para las estadísticas descriptivas. Se observó que había una diferencia significativa en las pruebas de flexibilidad antes y después del estiramiento estático. De este modo, se observó una mejora del $17 \%$ en el rendimiento promedio de la prueba con la aplicación de la agilidad de entrenamiento de estiramiento estático de los músculos isquiotibiales.

Palabras-Clave: Tenistas, elongacion Estático, Agilidad. 


\section{INTRODUÇÃO}

Os esportes de raquete veem ganho destaque e o número de pessoas praticante só aumenta. Por ser um dos esportes mais populares praticados com raquete, o tênis pode ser desfrutado por atletas ou jogadores recreativos de todas as idades e habilidades. ${ }^{1}$ Nos últimos anos esse esporte vem sofrendo mudanças, principalmente nas exigências físicas cada vez maiores, melhora nos níveis de treinamento e performance. ${ }^{2}$

Em decorrência das características do jogo, o desempenho do tenista durante uma partida exige habilidades físicas que lhe permitam paradas bruscas, acelerações e mudanças de direção. ${ }^{3}$ Desse modo, os membros inferiores são solicitados em diversas situações típicas durante a partida e as valências físicas como agilidade, força muscular e flexibilidade se manifestam nas suas diferentes formas, sendo o músculo ísquio-tibial muito solicitado nesse esporte. $^{4}$

Uma atenção especial deve ser dada ao grupo muscular ísquio-tibial, uma vez que o seu encurtamento é o mais encontrado na literatura, ${ }^{5}$ sendo o alongamento uma forma terapêutica de manutenção de níveis saudáveis deste grupamento muscular ${ }^{6}$. O alongamento pode ser aplicado no aquecimento ou em sessões específicas do treinamento com o intuito de aumentar a flexibilidade, diminuir a dor ao longo do movimento ${ }^{7}$ e redução das lesões musculares ${ }^{6}$. Apesar dos benefícios relacionados pouco se sabe sobre o efeito do alongamento sobre os movimentos de velocidade e precisão. ${ }^{8}$ No âmbito científico controvérsias sobre o alongamento muscular que antecede o exercício físico e o desempenho muscular são comuns ${ }^{2}$ e em especial nas atividades que sobrecarregam uma determinada musculatura, ou seja, atividades que exigem paradas bruscas, acelerações e mudanças de direção, como é o caso do tênis de campo., ${ }^{9} 10$

Desta forma há uma escassez de pesquisas em tenistas que comparam os efeitos agudos do alongamento estático durante o aquecimento e a associação com a valência agilidade, assim gerando indagações de como preparar uma sessão de treinamento haja vista as controvérsias quanto aos desfechos durante a prática, os quais podem proporcionar efeitos agudos prejudiciais ou benéficos em avaliações motoras subsequentes. ${ }^{11}$ Sendo assim, o presente estudo objetivou identificar se efeito agudo do alongamento estático dos músculos ísquios-tibiais influencia sobre a agilidade do tenista. 


\section{MÉTODOS}

\section{Amostra}

A pesquisa intencional foi composta de 10 tenistas que praticam o esporte Tênis de campo três vezes por semana, com duração média de 2 horas de atividade, todos do sexo masculino, com idade média de 32,8 $\pm 2,09$ anos, os mesmos não apresentavam familiarização com o teste de agilidade.

Os participantes da pesquisa foram previamente detectados com encurtamento dos músculos ísquios-tibais, sendo que só participava da pesquisa os avaliados que conseguissem obter o ângulo menor que $80^{\circ}$, os atletas que apresentassem uma angulação maior que $80^{\circ}$ foram excluídos da amostra.

\section{Protocolo}

\section{Amplitude articular}

A amplitude do movimento articular foi avaliada através de um goniômetro. De acordo com Polachini et al. ${ }^{12}$ não existem diferenças entre três métodos de avaliação de encurtamento de musculatura posterior da coxa. Portanto, utilizou-se no presente estudo um dos métodos propostos por Polachini e colaboradores ${ }^{12}$ que foi o teste de elevação do membro inferior estendido. Utilizou-se o protocolo de goniometria do Laboratório de Biometria e Fisiologia do Esforço (LABIFIE), da Universidade Federal Rural do Rio de Janeiro (UFRJ). ${ }^{13}$

O avaliado deveria estar deitado em decúbito dorsal, com as pernas estendidas. O goniômetro foi colocado com o seu eixo central sobre o ponto trocantérico, umas das hastes fixadas na parte lateral do tronco, sobre o prolongamento da linha axilar, e a outra face externa da coxa em sua linha mediana. Em seguida realizou-se a flexão da articulação do quadril. ${ }^{13}$

Para saber o comprimento dos músculos posteriores da coxa, utilizou-se o teste de elevação do membro inferior estendido, proposto por Kendall et al. ${ }^{14}$

Decúbito dorsal com os membros inferiores estendidos e a região lombar e o sacro achatado sobre a mesa. A padronização do teste requer que os joelhos estejam estendidos e 
que a região lombar e a pelve tenham uma posição fixa para controlar as variáveis criadas pela inclinação pélvica anterior ou posterior. Quando a região lombar e o sacro estão apoiados, manter uma coxa firmemente para baixo, usando a restrição passiva dos músculos flexores do quadril para evitar a inclinação pélvica posterior excessiva antes de iniciar a elevação do outro membro inferior. Com a região lombar e o sacro apoiado sobre a mesa e um membro inferior mantido firmemente para baixo, solicitar ao indivíduo que eleve o outro membro com o joelho estendido e o pé relaxado. O joelho é mantido estendido para controlar essa variável. O pé é mantido relaxado para evitar o envolvimento do músculo gastrocnêmico no nível do joelho. Quando o gastrocnêmico está contraído, a flexão dorsal do pé provoca flexão do joelho e, consequentemente, interfere no teste dos músculos posteriores da coxa se o joelho começar a se flexionar, baixar discretamente o membro inferior e solicitar ao indivíduo que estenda totalmente o joelho e eleve novamente o membro inferior, até sentir certa restrição e um leve desconforto. $O$ encurtamento é detectado quando se faz a flexão da coxa em direção à pelve e o ângulo em relação à mesa é menor que $80^{\circ} .{ }^{14}$

Após a detecção de encurtamento da musculatura dos ísquios-tibiais de ambos os membros inferiores, realizou-se 3 séries de alongamento usando a escala Perflex de esforço percebido na flexibilidade proposta por Dantas et al. ${ }^{15}$

Segundo Kendall et al. ${ }^{14}$ o exercício de alongamento com elevação do membro inferior estendido poder ser passivo ou ativo assistido. No presente estudo utilizou-se o método passivo, em que o indivíduo deve deitar-se na mesa com os membros inferiores estendidos e contar com a ajuda de outra pessoa para manter o membro que está sobre a mesa estendido e gradualmente elevar o outro membro com o joelho estendido.

De acordo com Milazzotto et al. ${ }^{6}$ três séries de alongamentos, mantidos por 30 segundos são suficientes para causar mudanças no comprimento muscular, sem que seja necessário mantê-lo por mais tempo. Tempos adicionais não trazem benefícios na ativação dos componentes musculares envolvidos no aumento da flexibilidade.

A Escala de Esforço Percebido na Flexibilidade - PERFLEX (FIGURA1) possui cinco níveis de intensidade, variando de 0 a 110, categorizados em cinco descritores verbais, para 
que o avaliando possa discernir, através da descrição da sua percepção, qual a sensação correspondente à amplitude de movimento realizado: de 0 a 30 - "normalidade"; de 31 a 60 - "forçamento"; de 61 a 80 - "desconforto"; de 81 a 90 - "dor suportável"; e de 91 a 110 - "dor forte". 13

\begin{tabular}{|c|c|c|c|}
\hline Nível & Descrição da sensação & Efeito & Especificação \\
\hline $0-30$ & normalidade & mobilidade & $\begin{array}{c}\text { não ocorre qualquer tipo de alteração em relação aos com- } \\
\text { ponentes mecânicos, componentes plásticos e componentes } \\
\text { inextensíveis. }\end{array}$ \\
\hline $31-60$ & forçamento & alongamento & $\begin{array}{c}\text { provoca deformação dos componentes plásticos e os compo- } \\
\text { nentes elásticos são estirados ao nível submáximo. }\end{array}$ \\
\hline $61-80$ & desconforto & flexionamento & $\begin{array}{c}\text { provoca adaptações duradouras nos componentes plásticos, } \\
\text { elásticos e inextensíveis. }\end{array}$ \\
\hline $81-90$ & dor suportável & possibilidade de lesão & $\begin{array}{c}\text { as estruturas músculo-conjuntivas envolvidas são submetidas } \\
\text { a um estiramento extremo, causando dor. }\end{array}$ \\
\hline $91+110$ & dor forte & lesão & $\begin{array}{c}\text { ultrapassa o estiramento extremo das estruturas envolvidas } \\
\text { incidindo, principalmente, sobre as estruturas esqueléticas. }\end{array}$ \\
\hline
\end{tabular}

Figura 1: Escala de esforço percebido na flexibilidade - PERFLEX

Fonte: DANTAS et al. ${ }^{13}$

\section{Teste de agilidade}

Para verificar o nível de agilidade dos tenistas executou-se o teste de Shuttle-Run, proposto por Dantas ${ }^{16}$ e foi classificado de acordo com Escores em percentis e desempenho proposto por Mathews, ${ }^{17}$ teste realizado antes e depois do treino de alongamento estático dos músculos ísquios-tibiais.

\section{Antropometria}

O IMC também foi avaliado para a caracterização da amostra onde foi coletado o peso corporal e a estatura. ${ }^{18}$ Para determinação do peso corporal foi utilizada uma balança digital da marca Plenna, com graduação de 100 gramas, e escalas, variando de 0 a $180 \mathrm{Kg}$. Para esta avaliação, os atletas estavam com vestimentas leves e sem o uso de qualquer calçado. ${ }^{19}$ A estatura foi verificada através de uma trena flexível marca Sanny Medical Sparrett, e precisão, de 0,1 mm fixada na parede lisa, com 3 metros e graduação de $0,1 \mathrm{~cm}$, e o zero coincidindo com o solo. O avaliado deveria estar na posição ortostática, em pé, posição ereta, braços estendidos ao longo do corpo, pés unidos, com os calcanhares em contato com o instrumento de medida, cintura pélvica, cintura escapular e região occipital. A medida foi realizada em apneia inspiratória, com a cabeça no plano de Frankfurt, paralela ao solo. ${ }^{20}$ Foi utilizado o esquadro antropométrico para verificar a perpendicularidade na coleta de dados. A partir dos dados antropométricos foi calculado o 
IMC e classificado de acordo com a (WHO/OMS $)^{21}$ e utilizou - se seguinte equação: IMC $=$ peso corporal $(\mathrm{kg}) / \operatorname{altura}^{2}(\mathrm{~m})$.

\section{Procedimento estatístico}

Para análise dos dados utilizada foi à estatística descritiva: média, desvio padrão, frequência de percentual (\%), e foi utilizado o teste T de Studant para verificar a diferença entre as médias á nível de significância $(\mathrm{p} \leq 0,05)$. Os dados foram analisados através do pacote estatístico BioEstat 5.0.

\section{RESULTADOS E DISCUSSÕES}

Na Tabela 1 estão apresentados os dados antropométricos dos avaliados a para caracterização da amostra.

Tabela 1 - Caracterizações das variáveis antropométricas da amostra

\begin{tabular}{ccc}
\hline Variáveis & Média & Desvio Padrão \\
\hline Peso Corporal & $83,7 \mathrm{~kg}$ & $\pm 7,76$ \\
Estatura & $1,82 \mathrm{~m}$ & $\pm 6,74$ \\
IMC & 25,18 & $\pm 1,78$ \\
\hline
\end{tabular}

De acordo com a WHO/OMS ${ }^{21}$ a população estudada está variando entre peso ideal e levemente acima do peso. Os resultados encontrados no teste de flexão da coxa em direção à pelve dos músculos ísquios-tibiais dos participantes do estudo estão apresentados na Tabela 2.

Tabela 2 - Comparação do encurtamento da flexão da coxa em direção à pelve dos tenistas avaliados

\begin{tabular}{cccc}
\hline $\begin{array}{c}\text { Flexão da coxa em } \\
\text { direção à pelve Direito }\end{array}$ & $\begin{array}{c}\text { Flexão da coxa em } \\
\text { direção à pelve Esquerda }\end{array}$ & t & p \\
\hline $67,8 \pm 10,12$ & $68,9 \pm 7,91$ & 0,27 & 0,39 \\
\hline
\end{tabular}

Podemos verificar na tabela acima a média da amplitude de movimento da flexão da coxa não diferenciou significativamente entre os hemisférios. Os resultados dos testes de ShutleRun antes e apos a intervenção com alongamento dos ísquios-tibiais estão dispostos na Tabela 3. 
Tabela 3 - Resultados de pré e pós teste de agilidade (Shuttle-Run)

\begin{tabular}{cccc}
\hline $\begin{array}{c}\text { Pré-teste sem } \\
\text { alongamento (s) }\end{array}$ & $\begin{array}{c}\text { Pós-teste com } \\
\text { alongamento (s) }\end{array}$ & t & p \\
\hline $10,74 \pm 0,88$ & $10,20 \pm 0,59$ & 1,78 & $0,04^{*}$ \\
\hline$* \mathrm{p}<0,05$ & & &
\end{tabular}

Na Tabela 3 é possível verificar que houve diferença estatística entre o teste de agilidade pré e pós-teste, após ser realizado o método de alongamento. A Tabela 4 apresenta o escore de percentil da agilidade encontrado no pré e pós-teste classificadas de acordo com os escores de desempenho do teste Shuttle Run. ${ }^{17}$

Tabela 4 - Comparação dos escores de percentil encontrado no pré e pós-teste de agilidade

\begin{tabular}{cccc}
\hline $\begin{array}{c}\text { Escore em percentis (\%) } \\
\text { pré-teste }\end{array}$ & $\begin{array}{c}\text { Escore em percentis (\%) } \\
\text { pós-teste }\end{array}$ & t & p \\
\hline $64,4 \pm 27,60$ & $81,6 \pm 14,59$ & $-1,741$ & $0,04^{*}$ \\
\hline$* \mathrm{p}<0,05$ & & &
\end{tabular}

Através dados apresentados na Tabela 4, pode-se perceber que após a realização do alongamento estático, ocorreu uma melhora de aproximadamente $17 \%$, de acordo com o escore de desempenho do teste de agilidade. Essa melhora ocorreu pelo motivo de o alongamento estático ser considerado o mais efetivo para aumentá-lo o nível de flexibilidade desse grupo muscular, justificando a teoria de que o alongamento estático lento facilita o disparo do órgão tendinoso de Golgi (OTG), que produz a inibição do músculo alongado. A explicação neurofisiológica sugere que o fator limitante durante o alongamento é a resistência muscular secundária a uma atividade reflexa. $\mathrm{O}$ mecanismo para aumentar a amplitude de movimento é as mudanças nas propriedades visco elásticas dos músculos. ${ }^{6}$ Provavelmente, além do alongamento ser efetivo na flexibilidade, o mesmo também pode ter relação com a agilidade.

Segundo Rubini ${ }^{22}$ existem poucas investigações sobre o efeito do alongamento no desempenho da agilidade. Contrapondo-se com o presente estudo Little e Williams ${ }^{23}$ pesquisaram o efeito do alongamento estático e do alongamento dinâmico em 18 jogadores profissionais de futebol e concluíram que o alongamento dinâmico teve benefícios na agilidade dos participantes do estudo, enquanto o alongamento estático não produziu mudanças no teste de agilidade. 
O mesmo aconteceu em estudo feito em 30 cadetes da United States Military Academy por McWillian et al., ${ }^{24}$ foi constatado que não houve diferença significativa no teste de ShuttleRun, com alongamento estático prévio, ou sem alongamento prévio. Em estudo realizado por Nelson e William ${ }^{25}$ os mesmos destacam que tanto alongamento estático quanto o alongamento dinâmico aumentam a flexibilidade do isquio-tibial quando comparado ao um grupo controle. Frabrício et al. ${ }^{2}$ destacam em seus achados que os do efeito agudo do alongamento estático passivo não promoveu alterações estatísticas significantes na força e potência muscular de atletas jovens de futebol.

No estudo de Gonçalves, Pavão e Dohnert ${ }^{7}$ com o objetivo de verificar os efeitos agudos e crônicos de um programa de alongamento estático em relação ao dinâmico na performance em jovens atletas de futebol. Os autores supracitados não encontraram diferenças significativas após intervenção aguda ou crônica nas valências impulsão vertical e agilidade. As duas formas de alongamento levaram a perda de rendimento no teste de velocidade e observou-se melhora da força muscular de isquio-tibiais ao longo do período do estudo em ambos os grupos avaliados. A atividade elétrica dos isquio-tibiais diminuiu significativamente na fase aguda para o grupo alongamento estático $(p=0,035)$ e aumentou significativamente na fase crônica no grupo alongamento dinâmico $(\mathrm{p}=0,038)$.

Carvalho et al. ${ }^{26}$ com intuito de foi verificar os efeitos agudos do alongamento estático e da facilitação neuromuscular proprioceptiva (FNP) sobre o desempenho de salto vertical em tenistas adolescentes, verificaram que os resultados não apresentaram mudança significativa na altura de salto $(\mathrm{p}=0,66)$ entre os testes pré e pós-alongamento estático e FNP. Os autores destacam que atletas que necessitam diretamente da força muscular para geração de potência, como os tenistas, parecem não ser beneficiados com exercícios de alongamento passivo nem FNP precedendo o exercício específico.

Ficou evidente que o alongamento agudo se mostrou benéfico na agilidade da população estudada, pois os avaliados não tinham familiarização com o teste de agilidade. Mas o tema exige estudos mais aprofundados, pois a melhora do resultado pode estar relacionada com a adaptação ao teste de Shuttle-Run. Não podemos extrapolar os resultados uma vez que a amostra foi de apenas 10 sujeitos e não utilizamos grupo controle. Acreditamos que há a 
necessidade de se realizar novos estudos com amostras maiores, e uso de grupo controle para a confirmação de nossos resultados.

\section{CONCLUSÃO}

Com base nos dados obtidos no estudo, pode-se observar que existiu uma diferença significativa entre pré e pós-teste de agilidade, com uma redução de $17 \%$ no escore percentilico.

\section{REFERÊNCIAS}

${ }^{1}$ GUEDES, J. M.; BARBIERI, D. F.; FIABANE, F. Lesões em tenistas competitivos. Revista Brasileira de Ciência do Esporte, Campinas, v. 31, n. 3, p. 217-229, 2010.

${ }^{2}$ FABRÍCIO, D. L. M. et al. Influência do alongamento estático agudo nas valências força e potência muscular em jovens futebolistas. Revista Fisioterapia e Saúde Funcional, Fortaleza, v. 1, n. 1, p. 4-9, 2012.

${ }^{3}$ KRAEMER, W. J. et al. Physiological changes with periodized resistance training in women tennis players. Medicine and Science in Sports and Exercise, Hagerstown, v. 35, n. 1, p. 157-168, 2003.

${ }^{4}$ EUCLYDES, P. T. et al. Qualidades físicas intervenientes e seu grau de importância no tênis de campo. Revista Mineira de Educação Física, Viçosa, v. 13, n. 1, p. 7-27, 2005.

${ }^{5}$ PINFILDI, C. E.; PRADO, R. P.; LIEBANO, R. E. Efeito do alongamento estático após diatermia de ondas curtas versus alongamento estático nos músculos isquiotibiais em mulheres sedentárias. Fisioterapia Brasil, Rio de Janeiro, v. 5, p. 119-24, 2005.

${ }^{6}$ MiLAZZOTTO, M. V.; CORAZZINA, L. G.; LIEBANO, R. E. Influencia do numero de series e tempo de alongamento estático sobre a flexibilidade dos músculos isquiotibiais em mulheres sedentárias. Revista Brasileira de Medicina no Esporte, v. 15, n. 6, 2009. 
${ }^{7}$ GONÇALVES, D. L.; PAVÃO, T. S.; DOHNERT, M. B. Efeitos agudos e crônicos de um programa no exercício e no esporte de alongamento estático e dinâmico no rendimento em jovens atletas do futebol. Revista Brasileira de Medicina no Esporte, São Paulo, v. 19, n. 4, 2013.

${ }^{8}$ KNUDSON, D. et al. Stretching has no effect on tennis serve performance. Journal of Strength \& Conditioning Research, Colorado Springs, v. 18, n. 3, 2004.

${ }^{9}$ SILVA, G. V. L. C. et al. Efeito agudo do alongamento estático sobre a força muscular isométrica. ConScientia e Saúde, v. 11, n. 2, p. 274-280, 2012.

${ }^{10} \mathrm{KUH}$, D. et al. Grip strength, postural control, and functional leg power in a representative cohort of British men and women: associations with physical activity, health status, and socioeconomic conditions. The Journal of Gerontology Series A: Biological Sciences and Medical Sciences, v. 60, p. 224-231, 2005.

${ }^{11}$ RIBEIRO, Y. S.; VECCHIO, F. B. D. Metanálise dos efeitos agudos do alongamento na realização de corridas curtas de alta intensidade. Revista Brasileira de Educação Física e Esporte, São Paulo, v. 25, n. 4, p. 567-581, 2011.

${ }^{12}$ POLACHINI, L. O. et al. Estudo comparativo entre três métodos de avaliação do encurtamento de musculatura posterior de coxa. Revista Brasileira de Fisioterapia, São Carlos, v. 9, n. 2, p. 187-193, 2005.

${ }^{13}$ DANTAS, E. H. M.; CARVALHO, J. L. T.; FONSECA, R. M. O. O protocolo LABIFIE de goniometria. Revista de Treinamento Desportivo, São Paulo, v. 2, n. 3, p. 21-34, 1997.

${ }^{10}$ KENDALL, F. P. et al. Músculos: provas e funções. 5. ed. São Paulo: Manole, 2007. 
${ }^{11}$ DANTAS, E. H. M. et al. Escala de esforço percebido na flexibilidade (PERFLEX): um instrumento adimensional para se avaliar a intensidade? Fitness Performance Jounal, Rio de Janeiro, v. 7, n. 5, p. 289-994, 2008.

${ }^{13}$ DANTAS, E. H. M. Flexibilidade, alongamento e flexionamento. 4. ed. Rio de Janeiro: Shape, 1998.

${ }^{14}$ MATHEWS, P. K. Medida e avaliação em educação física. 4. ed. Rio de Janeiro: Interamericana, 1980.

${ }^{15}$ POLLOCK, M.; WILMORE, J. H. Exercícios na saúde e na doença: avaliação e prescrição para prevenção e reabilitação. 2. ed. Rio de Janeiro: MEDSI, 1993.

${ }^{16}$ MONTEIRO, D. G.; FILHO, J. F. Comparação entre as características Somatotípicas o fracionamento da composição corporal em mulheres praticantes em academia de ginástica. Revista Brasileira de Ciência e Movimento, Brasília, v. 15, n. 1, p. 83-92, 2007.

${ }^{17}$ FERNANDES FILHO, J. A prática da avaliação física: testes, medidas e avaliação física em escolares, atletas e academias de ginástica. 2. ed. Rio de Janeiro: Shape, 2003.

${ }^{18}$ WORLD HEALTH ORGANIZATION (WHO/OMS). Disponível em: http://apps.who.int/bmi/index.jsp?introPage=intro_3.html. Acesso em: 10 out. 2013.

${ }^{19}$ RUBINI, E. C. Treinamento de flexibilidade: da teoria a prática. Rio de Janeiro: Sprint, 2010.

${ }^{20}$ LITTLE, T.; WILLIAMS, A. G. Effects of differential stretching protocols during warmups on high speed motor capacities in professional soccer players. Journal of Strength \& Conditioning Research, Colorado Springs, v. 20, n. 1, p. 203-207, 2006.

${ }^{21}$ MCWILLIAN, D. J. et al. Dynamics vs. static-stretching warm up: the effect on power and agility performance. Journal of Strength \& Conditioning Research, Colorado Springs, v. 20, n. 3, p. 492-499, 2006. 
${ }^{22}$ NELSON, R. T.; WILLIAM, D. B. Eccentric training and static stretching improve hamstring flexibility of high school males. Journal of Athletic Training, Dallas, v. 39, n. 3 p. $254-258,2004$.

${ }^{23}$ CARVALHO, F. L. P. et al. M. Efeitos agudos do alongamento estático e da facilitação neuromuscular proprioceptiva no desempenho do salto vertical de tenistas adolescentes. Fitness Performance Journal, Rio de Janeiro, v. 8, n. 4, p. 264-268, 2009. 